PROCEEDINGS OF THE

AMERICAN MATHEMATICAL SOCIETY

Volume 124, Number 8, August 1996

\title{
ON COMPACT CONNECTED SETS IN BANACH SPACES
}

\author{
F. S. DE BLASI AND J. MYJAK \\ (Communicated by Dale E. Alspach)
}

\begin{abstract}
Let $\mathbf{E}$ be a separable strictly convex Banach space of dimension at least 2 . It is shown that there exists a nonempty compact connected set $X \subset \mathbf{E}$ such that the nearest point mapping $p_{X}: \mathbf{E} \rightarrow 2^{\mathbf{E}}$ is not single valued on a set of points dense in E. Furthermore, it is proved that most (in the sense of the Baire category) nonempty compact connected sets $X \subset \mathbf{E}$ have the above property. Similar results hold for the furthest point mapping.
\end{abstract}

\section{INTRODUCTION}

Throughout this note $\mathbf{E}$ denotes a strictly convex Banach space with $\operatorname{dim} \mathbf{E} \geq 2$, and $2^{\mathbf{E}}$ the family of all nonempty subsets of $\mathbf{E}$. Additional assumptions about $\mathbf{E}$ will be made when needed. Set

$$
\mathcal{C}(\mathbf{E})=\left\{X \in 2^{\mathbf{E}} \mid X \text { is compact and connected }\right\} .
$$

The space $\mathcal{C}(\mathbf{E})$ is endowed with the Hausdorff distance $h$, under which it is a complete metric space.

For $X$ a nonempty compact subset of $\mathbf{E}$, we consider the nearest point mapping $p_{X}: \mathbf{E} \rightarrow 2^{\mathbf{E}}$ and the furthest point mapping $q_{X}: \mathbf{E} \rightarrow 2^{\mathbf{E}}$ defined, respectively, by

$p_{X}(a)=\{x \in X \mid\|x-a\|=d(a, X)\} \quad$ and $\quad q_{X}(a)=\{x \in X \mid\|x-a\|=e(a, X)\}$, where $d(a, X)=\inf \{\|x-a\| \mid x \in X\}$ and $e(a, X)=\sup \{\|x-a\| \mid x \in X\}$.

In a metric space $M$, an open (resp. closed) ball with center $x \in M$ and radius $r>0$ is denoted by $B_{M}(x, r)$ (resp. $\left.\widetilde{B}_{M}(x, r)\right)$.

By a result of Stečkin [2], it is known that for each nonempty compact set $X \subset \mathbf{E}$, $p_{X}$ is single valued on a residual subset of $\mathbf{E}$. On the other hand, Zamfirescu [3] has recently proved that for most (in the sense of the Baire category) nonempty compact sets $X \subset \mathbf{R}^{d}, d \geq 2$, the nearest point mapping $p_{X}$ is not single valued on a set of points dense in $\mathbf{R}^{d}$. In this note it is shown that the result of Zamfirescu remains valid in any strictly convex separable Banach space E. Furthermore, it is proved that for most nonempty compact connected sets $X \subset \mathbf{E}$, with $\mathbf{E}$ as before, the nearest point mapping is not single valued on a set of points dense in $\mathbf{E}$. Similar results hold for the furthest point mapping.

Received by the editors April 21, 1992

1991 Mathematics Subject Classification. Primary 47A52; Secondary 46B20, 54E52.

(C)1996 American Mathematical Society 


\section{MAIN RESUlts}

Theorem 1. Let $\mathbf{E}$ be a separable strictly convex Banach space, with $\operatorname{dim} \mathbf{E} \geq 2$. Let $\mathcal{C}_{0}$ be the set of all $X \in \mathcal{C}(\mathbf{E})$ such that the nearest point mapping $p_{X}: \mathbf{E} \rightarrow 2^{\mathbf{E}}$ is not single valued on a set of points dense in $\mathbf{E}$. Then $\mathcal{C}_{0}$ is a residual subset of $\mathcal{C}(\mathbf{E})$.

Proof. Let $A \subset \mathbf{E}$ be countable and dense in $\mathbf{E}$. For $k \in \mathbf{N}$, let $Q_{k}$ be the set of all rational numbers $r$ with $0<r<1 /(2 k)$. For $a \in A, k \in \mathbf{N}$ and $r \in Q_{k}$, put:

$$
\begin{aligned}
\mathcal{N}_{a} & =\{X \in \mathcal{C}(\mathbf{E}) \mid a \in X\}, \\
\mathcal{N}_{a, k} & =\{X \in \mathcal{C}(\mathbf{E}) \mid d(a, X)>1 / k\}, \\
\mathcal{N}_{a, k, r} & =\left\{X \in \mathcal{N}_{a, k} \mid p_{X} \text { is single valued at each point of } B_{\mathbf{E}}(a, r)\right\} .
\end{aligned}
$$

We claim that $\mathcal{N}_{a}$ is nowhere dense in $\mathcal{C}(\mathbf{E})$. Suppose the contrary. Since $\mathcal{N}_{a}$ is closed in $\mathcal{C}(\mathbf{E})$, there exist $X \in \mathcal{N}_{a}$ and $\varepsilon>0$ such that $B_{\mathcal{C}(\mathbf{E})}(X, \varepsilon) \subset \mathcal{N}_{a}$. If $a$ is an interior point of $X$ we fix $0<\tilde{\varepsilon}<\varepsilon$ so that $B_{\mathbf{E}}(a, \tilde{\varepsilon}) \subset X$ and define $Y=X \backslash B_{\mathbf{E}}(a, \tilde{\varepsilon} / 2)$. Since $Y \in \mathcal{C}(\mathbf{E}) \backslash \mathcal{N}_{a}$ and $h(Y, X)<\varepsilon$, a contradiction follows. If $a$ is in the boundary of $X$ we take $u \in \mathbf{E} \backslash X$ such that $0<\|u-a\|<\varepsilon / 2$ and define $Y=X+a-u$. Since $Y \in \mathcal{C}(\mathbf{E}) \backslash \mathcal{N}_{a}$ and $h(Y, X)<\varepsilon$, again a contradiction follows. Hence $\mathcal{N}_{a}$ is nowhere dense in $\mathcal{C}(\mathbf{E})$.

Claim. $\mathcal{N}_{a, k, r}$ is nowhere dense in $\mathcal{C}(\mathbf{E})$.

Postponing the proof of this claim, define

$$
\mathcal{N}=\left(\bigcup_{a \in A} \mathcal{N}_{a}\right) \cup\left(\bigcup_{a \in A} \bigcup_{k \in \mathbf{N}} \bigcup_{r \in Q_{k}} \mathcal{N}_{a, k, r}\right) .
$$

Clearly $\mathcal{N}$ is of the Baire first category in $\mathcal{C}(\mathbf{E})$. To complete the proof of the theorem it suffices to show that $\mathcal{C}(\mathbf{E}) \backslash \mathcal{N} \subset \mathcal{C}_{0}$.

Indeed, let $X \in \mathcal{C}(\mathbf{E}) \backslash \mathcal{N}$. Let $x \in \mathbf{E}$ and $\varepsilon>0$ be arbitrary. Let $a \in A \cap B_{\mathbf{E}}(x, \varepsilon)$. Let $k \in \mathbf{N}$ be such that $d(a, X)>1 / k$, thus $X \in \mathcal{N}_{a, k}$.

Take $r \in Q_{k}$ satisfying $0<r<\varepsilon-\|a-x\|$. Since $X \in \mathcal{N}_{a, k} \backslash \mathcal{N}_{a, k, r}$, there exists a point $u \in B_{\mathbf{E}}(a, r)$ such that $p_{X}(u)$ contains at least two different points. As $u \in B_{\mathbf{E}}(x, \varepsilon)$, and $x \in \mathbf{E}$ and $\varepsilon>0$ are arbitrary, it follows that $X \in \mathcal{C}_{0}$. Hence $\mathcal{C}(\mathbf{E}) \backslash \mathcal{N} \subset \mathcal{C}_{0}$ and $\mathcal{C}_{0}$ is residual in $\mathcal{C}(\mathbf{E})$, for $\mathcal{C}(\mathbf{E}) \backslash \mathcal{N}$ is so. This completes the proof.

Theorem 2. Let $\mathbf{E}$ be as in Theorem 1. Let $\mathcal{C}_{1}$ be the set of all $X \in \mathcal{C}(\mathbf{E})$ such that the furthest point mapping $q_{X}: \mathbf{E} \rightarrow 2^{\mathbf{E}}$ is not single valued on a set of points dense in $\mathbf{E}$. Then $\mathcal{C}_{1}$ is a residual subset of $\mathcal{C}(\mathbf{E})$.

Proof. Let $A \subset \mathbf{E}$ be countable and dense in $\mathbf{E}$. Let $Q^{+}$be the set of all strictly positive rationals. For $a \in A$ and $r \in Q^{+}$, define

$$
\begin{array}{r}
\mathcal{M}_{a, r}=\left\{X \in \mathcal{C}(\mathbf{E}) \mid X \backslash\{a\} \neq 0 \text { and } q_{X}\right. \text { is single valued } \\
\text { at each point of } \left.B_{\mathbf{E}}(a, r)\right\} .
\end{array}
$$

Claim. $\mathcal{M}_{a, r}$ is nowhere dense in $\mathcal{C}(\mathbf{E})$.

Postponing the proof of this claim, define

$$
\mathcal{M}=\left(\bigcup_{a \in A}\{a\}\right) \cup\left(\bigcup_{a \in A} \bigcup_{r \in Q^{+}} \mathcal{M}_{a, r}\right)
$$


From the claim and the fact that each set $\{a\}$, with $a \in A$, is nowhere dense in $\mathcal{C}(\mathbf{E})$, it follows that $\mathcal{C}(\mathbf{E}) \backslash \mathcal{M}$ is residual in $\mathcal{C}(\mathbf{E})$. Furthermore, as in the proof of Theorem 1, one can show that $\mathcal{C}(\mathbf{E}) \backslash \mathcal{M} \subset \mathcal{C}_{1}$. Hence also $\mathcal{C}_{1}$ is residual in $\mathcal{C}(\mathbf{E})$, completing the proof.

Set $\mathcal{K}(\mathbf{E})=\left\{X \in 2^{\mathbf{E}} \mid X\right.$ is compact $\}$. The space $\mathcal{K}(\mathbf{E})$ is endowed with the Hausdorff distance, under which it is a complete metric space.

The following Theorem 3 and Theorem 4 can be proved as Theorem 1 and Theorem 2, respectively. Theorem 3 generalizes a result obtained by Zamfirescu [3].

Theorem 3. Let $\mathbf{E}$ be as in Theorem 1. Let $\mathcal{K}_{0}$ be the set of all $X \in \mathcal{K}(\mathbf{E})$ such that the nearest point mapping $p_{X}: \mathbf{E} \rightarrow 2^{\mathbf{E}}$ is not single valued on a set of points dense in $\mathbf{E}$. Then $\mathcal{K}_{0}$ is a residual subset of $\mathcal{K}(\mathbf{E})$.

Theorem 4. Let $\mathbf{E}$ be as in Theorem 1. Let $\mathcal{K}_{1}$ be the set of all $X \in \mathcal{K}(\mathbf{E})$ such that the furthest point mapping $q_{X}: \mathbf{E} \rightarrow 2^{\mathbf{E}}$ is not single valued on a set of points dense in $\mathbf{E}$. Then $\mathcal{K}_{1}$ is a residual subset of $\mathcal{K}(\mathbf{E})$.

In view of Theorem 1 one has that most sets $X \in \mathcal{C}(\mathbf{E})$ are perfect and nowhere dense in $\mathbf{E}$. Moreover, from Theorem 3 and the fact that $\mathcal{C}(\mathbf{E})$ is nowhere dense in $\mathcal{K}(\mathbf{E})$, it follows that most sets $X \in \mathcal{K}(\mathbf{E})$ are perfect, not connected and nowhere dense in $\mathbf{E}$.

\section{Completion of the Proofs}

The following two lemmas are used in order to complete the proof of Theorem 1.

Lemma 1. Let $Z$ be a normed space. Let $a, u, v \in Z$ be such that $\|u-a\|=$ $\|v-a\|=r, u-a \neq-(v-a)$. Then, for every $\lambda \in[0,1]$,

$$
\left\|\frac{\lambda(u-a)+(1-\lambda)(v-a)}{\|\lambda(u-a)+(1-\lambda)(v-a)\|} r+a-u\right\| \leq\|v-u\| .
$$

Proof. It follows from Schäffer $[1$, p. 6].

Lemma 2. Let $Z$ be a normed space. Let $a, u \in Z, a \neq u$. For $t \in \mathbf{R}$, set $v_{t}=a+t(u-a)$. Then for every $x \in B_{\mathbf{E}}(a, r / 2)$, where $r=\|u-a\|$, and for every $t^{\prime}>t \geq 1$ we have

$$
\left\|v_{t}-x\right\| \leq\left\|v_{t^{\prime}}-x\right\| .
$$

Proof. In the contrary case there exist $x_{0} \in B_{\mathbf{E}}(a, r / 2)$ and $t_{1}>t_{0} \geq 1$ such that $\left\|v_{t_{1}}-x_{0}\right\|<\left\|v_{t_{0}}-x_{0}\right\|$. Clearly, $\left\|v_{t_{0}}-x_{0}\right\| \geq\left\|v_{t_{0}}-a\right\|-\left\|x_{0}-a\right\|>r-r / 2>\left\|x_{0}-a\right\|$. Let $0<s<1$ be such that $v_{t_{0}}=s v_{t_{1}}+(1-s) a$. Then

$$
\begin{aligned}
\left\|v_{t_{0}}-x_{0}\right\| & =\left\|s v_{t_{1}}+(1-s) a-x_{0}\right\| \\
& \leq s\left\|v_{t_{1}}-x_{0}\right\|+(1-s)\left\|a-x_{0}\right\| \\
& <s\left\|v_{t_{0}}-x_{0}\right\|+(1-s)\left\|v_{t_{0}}-x_{0}\right\|=\left\|v_{t_{0}}-x_{0}\right\|,
\end{aligned}
$$

a contradiction. This completes the proof.

To complete the proof of Theorem 1 it remains to show that the following claim is true.

Claim. $\mathcal{N}_{a, k, r}$ is nowhere dense in $\mathcal{C}(\mathbf{E})$. 
Proof. Indeed, let $X \in \mathcal{N}_{a, k, r}$ and $0<\varepsilon<\min \{r, \mu-1 / k\}$ be arbitrary, where $\mu=d(a, X)$. Note that $\varepsilon<\mu / 2$, for $\mu>1 / k>2 r>2 \varepsilon$. Using an idea from Zamfirescu [3], take $\tilde{y}_{1} \in X$ and $\tilde{y}_{2} \in \mathbf{E}$ satisfying $\left\|\tilde{y}_{i}-a\right\|=\mu, i=1,2$, and $\left\|\tilde{y}_{1}-\tilde{y}_{2}\right\|=\varepsilon / 8$. Observe that $\tilde{y}_{2}-a \neq-\left(\tilde{y}_{1}-a\right)$ since, in the contrary case, $\varepsilon / 8=\left\|\left(\tilde{y}_{1}-a\right)-\left(\tilde{y}_{2}-a\right)\right\|=2 \mu>4 \varepsilon$. Denote by $\gamma\left(\tilde{y}_{1}, \tilde{y}_{2}\right)$ the arc, with end points $\widetilde{y}_{1}$ and $\tilde{y}_{2}$, given by

$$
\gamma\left(\tilde{y}_{1}, \tilde{y}_{2}\right)=\left\{\frac{(1-\lambda)\left(\tilde{y}_{1}-a\right)+\lambda\left(\tilde{y}_{2}-a\right)}{\left\|(1-\lambda)\left(\tilde{y}_{1}-a\right)+\lambda\left(\tilde{y}_{2}-a\right)\right\|} \mu+a \mid \lambda \in[0,1]\right\} .
$$

This is well defined, for $\tilde{y}_{2}-a \neq-\left(\tilde{y}_{1}-a\right)$. Furthermore, for $i=1,2$, set

$$
y_{i}=\tilde{y}_{i}-\frac{\varepsilon}{4 \mu}\left(\tilde{y}_{i}-a\right), \quad u_{i}=a+\frac{\varepsilon}{8 \mu}\left(\tilde{y}_{i}-a\right),
$$

and define $Y=X \cup y_{1} \tilde{y}_{1} \cup y_{2} \tilde{y}_{2} \cup \gamma\left(\tilde{y}_{1}, \tilde{y}_{2}\right)$. Here $y_{i} \tilde{y}_{i}, i=1,2$, denotes the segment in $\mathbf{E}$ with end points $y_{i}$ and $\tilde{y}_{i}$. Clearly $Y \in \mathcal{N}_{a, k}$, because $Y \in \mathcal{C}(\mathbf{E})$ and $d(a, Y)=$ $\mu-\varepsilon / 4>1 / k$. If $y \in y_{1} \tilde{y}_{1} \cup y_{2} \tilde{y}_{2}$ then $d(y, X) \leq 3 \varepsilon / 8$, while if $y \in \gamma\left(\tilde{y}_{1}, \tilde{y}_{2}\right)$, by Lemma 1 one has $d(y, X) \leq\left\|\tilde{y}_{1}-\tilde{y}_{2}\right\|=\varepsilon / 8$. Hence $h(Y, X) \leq 3 \varepsilon / 8<\varepsilon / 2$.

Set

$$
\rho=\min \left\{\frac{\left\|y_{2}-u_{1}\right\|-\left\|y_{1}-u_{1}\right\|}{2}, \frac{\left\|y_{1}-u_{2}\right\|-\left\|y_{2}-u_{2}\right\|}{2}, \frac{1}{3} \delta\left(y_{1} \tilde{y}_{1}, y_{2} \tilde{y}_{2}\right), \frac{\varepsilon}{16}\right\},
$$

where $\delta\left(y_{1} \tilde{y}_{1}, y_{2} \tilde{y}_{2}\right)=\inf \left\{\left\|x_{1}-x_{2}\right\| \mid x_{1} \in y_{1} \tilde{y}_{1}, x_{2} \in y_{2} \tilde{y}_{2}\right\}$. Observe that $\rho>0$, because $\mathbf{E}$ is strictly convex and $\delta\left(y_{1} \tilde{y}_{1}, y_{2} \tilde{y}_{2}\right)>0$. Furthermore, $B_{\mathcal{C}(\mathbf{E})}(Y, \rho) \subset$ $\mathcal{N}_{a, k}$, since each $Z \in B_{\mathcal{C}(\mathbf{E})}(Y, \rho)$ satisfies $d(a, Z) \geq d(a, Y)-h(Z, Y)>\mu-\varepsilon / 4-\rho>$ $\mu-\varepsilon>1 / k$.

Let $Z \in B_{\mathcal{C}(\mathbf{E})}(Y, \rho)$. Set

$$
\widetilde{Z}=Z \cap \widetilde{B}_{\mathbf{E}}\left(a,\left\|y_{1}-a\right\|+\rho\right), \quad \widetilde{Z}_{i}=\widetilde{Z} \cap\left(y_{i} \tilde{y}_{i}+\rho \widetilde{B}\right), \quad i=1,2,
$$

where $\widetilde{B}=\widetilde{B}_{\mathbf{E}}(0,1)$. We have $\widetilde{Z}_{i} \neq \varnothing, i=1,2, \widetilde{Z}_{1} \cap \widetilde{Z}_{2}=\varnothing, \widetilde{Z}_{1} \cup \widetilde{Z}_{2}=\widetilde{Z}$.

Indeed, for $i=1,2, y_{i} \in Y \subset Z+\rho \widetilde{B}$; thus, for some $z_{i} \in Z,\left\|z_{i}-y_{i}\right\| \leq \rho$, which implies $\left\|z_{i}-a\right\| \leq\left\|y_{i}-a\right\|+\rho$. As $z_{i} \in \widetilde{Z}_{i}, i=1,2$, the sets $\widetilde{Z}_{1}$ and $\widetilde{Z}_{2}$ are nonempty. Let us show that $\widetilde{Z}_{1} \cap \widetilde{Z}_{2}=\varnothing$. Supposing otherwise, let $z \in \widetilde{Z}_{1} \cap \widetilde{Z}_{2}$. Then, for some $v_{i} \in y_{i} \tilde{y}_{i}$ we have $\left\|v_{i}-z\right\| \leq \rho, i=1,2$, and so $\delta\left(y_{1} \tilde{y}_{1}, y_{2} \tilde{y}_{2}\right) \leq$ $\left\|v_{1}-v_{2}\right\| \leq\left\|v_{1}-z\right\|+\left\|v_{2}-z\right\| \leq 2 \rho$, a contradiction. Hence $\widetilde{Z}_{1} \cap \widetilde{Z}_{2}=\varnothing$. It remains to prove that $\widetilde{Z}_{1} \cup \widetilde{Z}_{2}=\widetilde{Z}$. To this end, let $z \in \widetilde{Z}$. Then $z \in Z$ and $\|z-a\| \leq\left\|y_{1}-a\right\|+\rho \leq \mu-\varepsilon / 4+\varepsilon / 16=\mu-3 \varepsilon / 16$. Since $z \in Z \subset Y+\rho \widetilde{B}$, for some $y \in Y$ we have $\|z-y\| \leq \rho$. If $y \in X \cup \gamma\left(\tilde{y}_{1}, \tilde{y}_{2}\right)$, then $\|y-a\| \geq \mu$ and so

$$
\rho \geq\|z-y\| \geq\|y-a\|-\|z-a\| \geq \mu-\left(\mu-\frac{3 \varepsilon}{16}\right)=\frac{3 \varepsilon}{16},
$$

a contradiction to equality (3.1). Hence $y \in y_{1} \tilde{y}_{1} \cup y_{2} \tilde{y}_{2}$. Since $z=y+(z-y)$ $\in\left(y_{1} \tilde{y}_{1}+\rho \widetilde{B}\right) \cup\left(y_{2} \tilde{y}_{2}+\rho \widetilde{B}\right)$ and $z \in \widetilde{Z}$ is arbitrary, it follows that $\widetilde{Z} \subset \widetilde{Z}_{1} \cup \widetilde{Z}_{2}$. The reverse inclusion being trivial, we have $\widetilde{Z}_{1} \cup \widetilde{Z}_{2}=\widetilde{Z}$.

Now let $u \in u_{1} u_{2}$ be arbitrary. We have

$$
d(u, Z)<d\left(u, Y_{1}\right),
$$


where $Y_{1}=X \cup\left(\tilde{y}_{1}, \tilde{y}_{2}\right)+\rho \widetilde{B}$. In order to prove (3.2) take $z_{1} \in Z$ such that $\left\|y_{1}-z_{1}\right\| \leq \rho$. Clearly,

$$
\left\|u-z_{1}\right\| \leq\left\|u-u_{1}\right\|+\left\|u_{1}-y_{1}\right\|+\left\|y_{1}-z_{1}\right\| \leq\left\|u_{1}-u_{2}\right\|+\left(\mu-\frac{3 \varepsilon}{8}\right)+\rho .
$$

Furthermore, $\left\|u_{1}-u_{2}\right\|=\varepsilon\left\|\tilde{y}_{1}-\tilde{y}_{2}\right\| /(8 \mu)=\varepsilon^{2} /(64 \mu)<\varepsilon / 8$, for $\varepsilon<\mu$. Hence $\left\|u-z_{1}\right\|<\mu-\varepsilon / 4+\rho$, which yields

$$
d(u, Z)<\mu-\frac{\varepsilon}{4}+\rho .
$$

On the other hand, let $y \in Y_{1}$ be arbitrary. Then $y=x+v$, for some $x \in$ $X \cup \gamma\left(\tilde{y}_{1}, \tilde{y}_{2}\right)$ and $v \in \rho \widetilde{B}$. As $\|u-y\| \geq\|x-a\|-\|u-a\|-\|v\| \geq \mu-\varepsilon / 8-\rho$, we have

$$
d\left(u, Y_{1}\right) \geq \mu-\frac{\varepsilon}{8}-\rho .
$$

Combining (3.3) and (3.4) gives (3.2), since $\mu-\varepsilon / 8-\rho \geq \mu-\varepsilon / 4+\rho$.

By virtue of (3.3), each $z \in p_{Z}(u)$ satisfies $\|u-z\|<\mu-\varepsilon / 4+\rho=\left\|y_{1}-a\right\|+\rho$; thus $z \in \widetilde{Z}$. Furthermore, (3.2) implies $p_{Z}(u) \cap Y_{1}=\varnothing$, from which, in view of the definition of $Y$, it follows that $p_{Z}(u) \subset\left(y_{1} \tilde{y}_{1}+\rho \widetilde{B}\right) \cup\left(y_{2} \tilde{y}_{2}+\rho \widetilde{B}\right)$. Hence

$$
p_{Z}(u) \subset \widetilde{Z}_{1} \cup \widetilde{Z}_{2} .
$$

Let $z \in \widetilde{Z}_{2}$ be arbitrary. Let $y \in y_{2} \tilde{y}_{2}$ be such that $\|y-z\| \leq \rho$. By Lemma 2 , $\left\|y-u_{1}\right\| \geq\left\|y_{2}-u_{1}\right\|$. Since $\left\|u_{1}-z\right\| \geq\left\|u_{1}-y\right\|-\rho \geq\left\|u_{1}-y_{2}\right\|-\rho$, we have

$$
\left\|u_{1}-y_{2}\right\| \leq d\left(u_{1}, \widetilde{Z}_{2}\right)+\rho .
$$

Clearly $d\left(u_{1}, \widetilde{Z}_{1}\right) \leq\left\|u_{1}-y_{1}\right\|+\rho$, for $\widetilde{Z}_{1} \cap B_{\mathbf{E}}\left(y_{1}, \rho\right) \neq \varnothing$. Moreover, $\left\|u_{1}-y_{1}\right\| \leq$ $\left\|u_{1}-y_{2}\right\|-2 \rho$, by virtue of the definition of $\rho$. Thus, $d\left(u_{1}, \widetilde{Z}_{1}\right) \leq\left\|u_{1}-y_{2}\right\|-\rho$. Combining this with (3.5) gives

$$
d\left(u_{1}, \widetilde{Z}_{1}\right) \leq d\left(u_{1}, \widetilde{Z}_{2}\right) .
$$

Analogously one can show that $d\left(u_{2}, \widetilde{Z}_{1}\right) \geq d\left(u_{2}, \widetilde{Z}_{2}\right)$. From this, (3.6) and the continuity of the function $u \rightarrow d\left(u, \widetilde{Z}_{1}\right)-d\left(u, \widetilde{Z}_{2}\right)$ from $u_{1} u_{2}$ to $\mathbf{R}$, it follows that there exists at least one point $u \in u_{1} u_{2}$ such that $d\left(u, \widetilde{Z}_{1}\right)=d\left(u, \widetilde{Z}_{2}\right)$. Furthermore, it is easy to see that $d\left(u, \widetilde{Z}_{i}\right)=d(u, Z), i=1,2$, and so $p_{\widetilde{Z}_{i}}(u) \subset p_{Z}(u), i=1,2$. Consequently, $p_{Z}(u)$ contains at least two different points, since $\widetilde{Z}_{1} \cap \widetilde{Z}_{2}=\varnothing$. Hence $Z \in \mathcal{N}_{a, k} \backslash \mathcal{N}_{a, k, r}$. Since $Z$ is arbitrary in $B_{\mathcal{C}(\mathbf{E})}(Y, \rho)$, it follows that $B_{\mathcal{C}(\mathbf{E})}(Y, \rho) \cap$ $\mathcal{N}_{a, k, r}=\varnothing$, completing the proof.

To complete the proof of Theorem 2 it remains to show that the following claim is true.

Claim. $\mathcal{N}_{a, r}$ is nowhere dense in $\mathcal{C}(\mathbf{E})$.

Proof. Indeed, let $X \in \mathcal{M}_{a, r}$ be arbitrary. Let $0<\varepsilon<\min \{r, \nu\}$, where $\nu=$ $e(a, X)$. Let $\tilde{y}_{1} \in X$ and $\tilde{y}_{2} \in \mathbf{E}$ satisfy $\left\|\tilde{y}_{i}-a\right\|=\nu, i=1,2$, and $\left\|\tilde{y}_{i}-\tilde{y}_{2}\right\|=\varepsilon / 4$. For $i=1,2$, set

$$
y_{i}=\tilde{y}_{i}+\frac{\varepsilon}{2 \nu}\left(\tilde{y}_{i}-a\right), \quad u_{i}=a+\frac{\varepsilon}{8 \nu}\left(\tilde{y}_{i}-a\right),
$$

and define $Y=X \cup y_{1} \tilde{y}_{1} \cup y_{2} \tilde{y}_{2} \cup \tilde{y}_{1} \tilde{y}_{2}$. Clearly, $Y \in \mathcal{C}(\mathbf{E})$ and $h(Y, X) \leq 3 \varepsilon / 4$. 
Let $Z \in B_{\mathcal{C}(\mathbf{E})}(Y, \rho)$ be arbitrary, where $\rho$ is given by (3.1). Set

$$
Z_{i}=Z \cap\left(\tilde{y}_{i} y_{i}+\rho \widetilde{B}\right), \quad i=1,2 .
$$

It is evident that $Z_{1}, Z_{2} \neq \varnothing$. Furthermore, $Z_{1} \cap Z_{2}=\varnothing$. Supposing otherwise, let $z \in Z_{1} \cap Z_{2}$. Then for some $v_{i} \in y_{i} \tilde{y}_{i}, i=1,2$, we have $\left\|z-v_{i}\right\| \leq \rho, i=1,2$, and so $3 \rho \leq \varepsilon\left(y_{1} \tilde{y}_{1}, y_{2} \tilde{y}_{2}\right) \leq\left\|v_{1}-v_{2}\right\| \leq 2 \rho$, a contradiction. Let $u \in u_{1} u_{2}$ be arbitrary. Since each $y \in Y_{1}=\left(X \cup \tilde{y}_{1} \tilde{y}_{2}\right)+\rho \widetilde{B}$ satisfies $\|u-y\| \leq\|y-a\|+\|u-a\| \leq$ $(\nu+\rho)+\varepsilon / 8$, we have

$$
e\left(u, Y_{1}\right) \leq \nu+\frac{\varepsilon}{8}+\rho .
$$

Let $z \in Z \cap B_{\mathbf{E}}\left(y_{1}, \rho\right)$. Clearly,

$$
\begin{aligned}
e(u, Z) & \geq\|u-z\| \geq\left\|u-y_{1}\right\|-\left\|z-y_{1}\right\| \\
& >\left\|u_{1}-y_{1}\right\|-\left\|u_{1}-u\right\|-\rho \geq\left\|u_{1}-y_{1}\right\|-\left\|u_{1}-u_{2}\right\|-\rho .
\end{aligned}
$$

Moreover, $\left\|u_{1}-y_{1}\right\|=\nu+3 \varepsilon / 8$, and $\left\|u_{1}-u_{2}\right\|=\varepsilon\left\|\tilde{y}_{1}-\tilde{y}_{2}\right\| /(8 \nu)=\varepsilon^{2} /(32 \nu)<\varepsilon / 8$, since $\varepsilon<\nu$. Hence,

$$
e(u, Z)>\nu+\frac{\varepsilon}{4}-\rho .
$$

By virtue of (3.7) and (3.8), observing that $\nu+\varepsilon / 8+\rho<\nu+\varepsilon / 4-\rho$, it follows that

$$
e\left(u, Y_{1}\right)<e(u, Z) \text {. }
$$

This implies $q_{Z}(u) \cap Y_{1}=\varnothing$ and so, from the definition of $Y, q_{Z}(u) \subset Z_{1} \cup Z_{2}$.

Let $z \in Z_{1}$. Let $y \in y_{1} \tilde{y}_{1}$ be such that $\|y-z\| \leq \rho$. Using (3.1), we have

$$
\begin{aligned}
\left\|u_{1}-z\right\| & \leq\left\|u_{1}-y\right\|+\|y-z\| \leq\left\|u_{1}-y_{1}\right\|+\rho \leq\left\|u_{1}-y_{2}\right\|-\rho \\
& \leq\left\|u_{1}-v\right\|+\left\|v-y_{2}\right\|-\rho<\left\|u_{1}-v\right\| \leq e\left(u_{1}, Z_{2}\right),
\end{aligned}
$$

for some $v \in Z_{2} \cap B_{\mathbf{E}}\left(y_{2}, \rho\right)$, and so, since $z \in Z_{1}$ is arbitrary,

$$
e\left(u_{1}, Z_{1}\right) \leq e\left(u_{1}, Z_{2}\right) .
$$

Likewise one can show that $e\left(u_{2}, Z_{1}\right) \geq e\left(u_{2}, Z_{2}\right)$. Then, arguing as in the proof of the preceding claim, one proves that $\mathcal{M}_{a, r}$ is nowhere dense in $\mathcal{C}(\mathbf{E})$. This completes the proof.

\section{REFERENCES}

1. J. J. Schäffer, Geometry of spheres in normed spaces, Dekker, New York, 1976. MR 57:7120

2. S. B. Stečkin, Approximation properties of sets in normed linear spaces, Rev. Roumaine Math. Pures Appl. 8 (1963), pp. 5-13. MR 27:5018

3. T. Zamfirescu, The nearest point mapping is single valued nearly everywhere, Arch. Math. (Basel) 51 (1990), pp. 563-566. MR 91k:41061

Dipartimento di Matematica, Università di Roma II (Tor Vergata), Via della Ricerca SCientifica, 00133 Roma, Italy

Dipartimento di Matematica, Università di L'Aquila, Via Vetoio, 67100 L'Aquila, ITALY

E-mail address: myjak@axscaq.aquila.imtn.it 